\title{
An experimental study on the effects of exposure to magazine advertising on children's food choices
}

\author{
Sandra C Jones ${ }^{1, *}$ and Lisa Kervin ${ }^{2}$ \\ ${ }^{1}$ Centre for Health Initiatives, University of Wollongong, Room 41.G04, Wollongong, NSW 2522, Australia: \\ ${ }^{2}$ Faculty of Education, University of Wollongong, Wollongong, NSW, Australia
}

Submitted 1 April 2010: Accepted 23 September 2010: First published online 8 December 2010

\begin{abstract}
Objective: The present study sought to determine the feasibility of an experimental research design to investigate the effects of exposure to magazine advertising on children's food choices.

Design: Children were randomized to read either a magazine with food advertisements or a magazine with no food advertisements. They then chose two food items from the intervention 'store' to eat after the session. Data were also collected on attitudes to advertising and snack food preferences. Finally, participants' parents were provided with a self-completion survey on food choices and other variables ( $n$ 24). Setting: Three vacation care centres in regional New South Wales, Australia.

Subjects: Children aged 5-12 years ( $n$ 47).

Results: Children in the experimental condition were more likely to choose advertised foods than those in the control group. Interestingly, the majority reported taste and healthiness as the most important factors in snack food choices; however, when faced with the actual food choice, they predominantly chose unhealthy foods (eighty-two unhealthy and only twelve healthy items were chosen).

Conclusions: This was the first study to assess the effects on children of exposure to food advertising within the context of reading a child-targeted magazine. Importantly, even with the small sample size and venue limitations, we found that exposure to magazine advertising influenced food choices. Children's magazines are an underresearched and poorly regulated medium, with considerable potential to influence children's food choices. The present study shows that the methodology is feasible, and future studies could replicate this with larger samples.
\end{abstract}

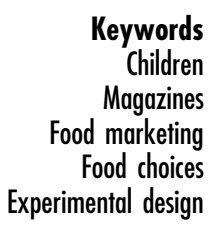

Children's food choices, and their consequences, are an issue of concern worldwide. In 2006, it was estimated that there were more than 21 million overweight or obese children in the European Union (about 30\% of all children), approximately one-quarter of whom are obese, and this figure is increasing by over 1 million children each year ${ }^{(1)}$. In the USA, it is estimated that $35 \%$ of children are overweight or obese ${ }^{(2)}$. Prevalence is increasing in nearly every country to the point that overnutrition rivals undernutrition as the chief food problem even in developing countries ${ }^{(3)}$. In Australia, the prevalence of overweight and obesity among children aged 7-16 years increased from 11\% in 1985 to $21 \%$ in $1995^{(4)}$ and, by $2005,25 \%$ of $5-17$-year-olds were classified as overweight $(17 \%)$ or obese $(8 \%)^{(5)}$.

Studies in the USA show that the majority of children consume insufficient amounts of fruit, vegetables and dietary fibre ${ }^{(6)}$ and higher than recommended amounts of energy-dense and high-fat foods ${ }^{(7)}$. Recent research confirms that children are eating outside the home more regularly, eating larger portion sizes, consuming more soft drinks and eating less fruit and vegetables ${ }^{(8)}$. In
Australia, the energy intake of children aged $10-15$ years increased by $12 \%$ for girls and by $15 \%$ for boys between 1985 and 1995, because of increased consumption of energy-dense, nutrient-poor foods and beverages ${ }^{(9)}$.

There is a raft of factors that influence children's eating patterns. These include, but are not limited to, direct parental influences (controls over food); indirect parental influences, such as parents' own consumption practices ${ }^{(10)}$ and nutrition knowledge ${ }^{(11)}$; environmental factors (such as food availability); and social and cultural influences ${ }^{(12)}$.

\section{Child-targeted food advertising}

Advertising (and particularly television (TV) advertising) has been argued to be an important factor in children's eating patterns. There is consistent and increasing evidence that food advertising targeted at children is predominantly for unhealthy foods. It has been estimated that children view in excess of 40000 TV advertisements each year ${ }^{(13)}$, with many of these being for food ${ }^{(14)}$. Studies on TV food 
advertising during children's viewing times have consistently found that advertisements are predominantly for foods high in fat, salt or sugar in the $\mathrm{USA}^{(15)}$, the $\mathrm{UK}^{(16)}$, Portugal $^{(17)}$, Iran ${ }^{(18)}$, New Zealand ${ }^{(19)}$ and Australia ${ }^{(20)}$.

A secondary analysis of a longitudinal survey data set in New Zealand found that children who watched more than $2 \mathrm{~h}$ of $\mathrm{TV} / \mathrm{d}$ were more than twice as likely to be frequent consumers of foods commonly advertised on TV, including soft drinks, hamburgers and French fries ${ }^{(21)}$. Similarly, a Dutch study found that children's exposure to food advertising was associated with greater consumption of advertised brands and of energy-dense foods more generally $^{(22)}$. On a positive note, a study across nine European countries concluded that exposure to TV advertisements for healthy foods was positively associated with reported fruit and vegetable intake ${ }^{(23)}$.

Hastings et $\mathrm{al}^{(16)}$ argue that the effect of food promotion has been understated because indirect effects have been ignored, and because the cumulative effect of TV advertising combined with other forms of promotion and marketing is likely to be greater. Further, the effects of advertising on children have considerable implications for families, with some evidence that children affect the buying patterns of parents by requesting, or even demanding, specific snacks and meal selections ${ }^{(14)}$. For example, a recent observational study of parent-child co-shoppers found that children made food requests in $50.4 \%$ of observations, and almost half of these requests were granted ${ }^{(24)}$. It has been argued that the consumption of consumer goods and services has become an important part of family leisure activities in industrialized, developed democracies and children are being raised in family settings where a dominant narrative is one of 'having more and responding to desire or needs immediately' (p. 601) ${ }^{(25)}$.

It appears that marketers are tapping into this family dynamics by appealing to both parents and children with different targeted messages that serve to increase both children's food requests and parents' willingness to meet these requests. An earlier study of Australian print food advertisements identified concurrent campaigns in children's magazines positioning food products as 'fun' and in women's magazines positioning them as 'nutritious ${ }^{,(44)}$. For example, $82 \%$ of adults shown the 'parent' version of Kellogg's LCM advertisement perceived the message to be that the product was healthy, compared with only $26 \%$ shown the 'child' version; concurringly, 56\% shown the 'parent' version and $78 \%$ shown the 'child' version also perceived the message that the product would make the purchaser/consumer popular.

\section{Experimental studies of the effects of child-targeted food advertising}

The impact of advertising on children's food choices has been shown both in econometric studies - which have concluded that increases in fast-food advertising were associated with increases in the proportion of overweight children $^{(26)}$, and that exposure to TV advertising is associated with obesity in children ${ }^{(27)}$ - and direct observational or experimental studies. A recent update of Hasting and colleagues ${ }^{\text {(28) }}$ review of the literature identified six experimental studies assessing the effect of exposure to TV advertising on children's consumption, including increased preference for less-healthy food choices, increased energy consumption and increased total food intake. However, they did not identify any studies on the effects of non-TV food advertising on children's food preferences and choices, despite the need for such research being a key recommendation of many reviews ${ }^{(16,29,30)}$.

\section{Print media food advertising}

To date, the focus of research on food advertising to children has been in the context of TV advertising. Thus, there is a solid and growing evidence base showing that TV food advertising increases children's preferences for, and consumption of, advertised foods ${ }^{(16,29-31)}$. Over 20 years ago, researchers cautioned that relying on TV advertising as the sole, or primary, medium for studying the influences of advertising on children resulted in an incomplete understanding of how children process and interpret advertising ${ }^{(32,33)}$.

The opportunity to reach children and teenagers with print media has expanded over the last decade ${ }^{(34)}$; in Australia, the number of magazines targeting children is increasing, and they are increasingly identifying and developing niche markets. For example, under the 'Disney' title, ACP magazines publishes: Disney Adventures, targeting children aged $\geq 6$ years; Disney Girl, which targets girls aged 6-13 years who are described as '(spending their) pocket money on lip glosses, toys, CDs, DVDs, clothing and accessories'; and Disney's Princess, which targets girls aged 3-9 years (http://www.acp.com.au/Magazines.htm). There are also magazines based on specific characters or entertainers - such as Barbie, Hannah Montana, High School Musical (all published by Otter Press) and The Wiggles (published by Next Media).

The 2009 update of the literature found that there had been several recent studies conducted on media other than TV, with a small number on magazines, websites or magazines and linked websites combined ${ }^{(28)}$. However, these studies tend to be descriptive in nature, focusing on the extent and content of such advertising ${ }^{(35,36)}$.

A recent study (published after the 2009 review) had 309 children read a booklet containing healthy food advertisements, unhealthy food advertisements or nonfood advertisements; they were then given a choice of a packet of raisins or a packet of sultanas ${ }^{(37)}$. No significant difference in food choices between the three groups were found. However, that study did not examine responses to 
food advertisements in a natural context but rather to advertisements in isolation, with children asked to read and answer questions about each advertisement, and preceded and followed (before the food choice) with 'current state' questions (which included whether they saw themselves as good looking, liked, hungry, healthy, fat, etc.). This raises the question as to whether the food choices were influenced by the artificial exposure and processing of the advertisements and with the priming of perceptions associated with food choice, and thus whether the same result would be found if the children interacted with advertising in a more natural manner.

\section{Method}

\section{Participants}

The participants in the present study were primaryschool-aged children attending vacation care programmes at three not-for-profit centres in regional New South Wales, Australia. Each centre operates within a public school, and provides supervised care for children aged 5-12 years during school holidays. All data were collected in January 2010.

\section{Stimuli}

The stimuli were the December 2009 issues of two topselling children's magazines. Krash has a publisherreported monthly circulation of $45000^{*}$ in Australia and 12000 in New Zealand (http://www.nuclear.com.au/ mediakits.html) and is positioned as 'The world's coolest boy's mag' (http://www.krash.com.au/); its core target consists of boys aged 8-14 years. D-Mag has an audited monthly circulation of 27126 ; it promises 'Fun, discovery and adventure' (http://www.dmag.com.au/) and targets boys and girls aged 6-13 years. Although we ideally would have liked to use two gender-neutral magazines, D-Mag was the only publication that does not have a single-gender focus. Our previous qualitative research suggested that girls would still find articles of interest in a 'boys' magazine but that boys would not be prepared to read a 'girls' magazine (such as Total Girl or Barbie). The Krash magazine contained a full-page advertisement for a range of candy products; D-Mag contained no food advertisements.

\section{Procedure}

The study procedure consisted of five phases (questionnaire 1; intervention, distracter task; questionnaire 2; purchase activity; and questionnaire 3). The study was conducted in the late morning in all three centres (approximately $2 \mathrm{~h}$ before lunch), and planned to ensure that the 'break' coincided with the scheduled morning teatime. This also meant that the participants were unlikely

\footnotetext{
* Krash is not audited by the Audit Bureau of Circulation.
}

to be still satiated from their breakfast, but to be moderately hungry.

Before exposure to the experimental stimuli, participants completed a brief questionnaire on their attitudes towards magazine advertising, factors that influence their snack food choices and demographic information (questionnaire 1). Participants were then randomly allocated to one of the two conditions - experimental (magazine with food advertisements) or control (magazine with no food advertisements) - and given $15 \mathrm{~min}$ to read the magazine at their own pace (intervention). They were not directed to read any particular section of the magazine, but simply to read it as they would at home - although they were encouraged to try to look at all the pages.

Participants then completed a distracter task, in which they were asked to read the 'most embarrassing moment' section in the magazine (both magazines have a page on which readers write in and tell of an embarrassing experience), choose one of the reader's stories and write down what advice they would give that person. They then completed a brief series of questions about magazines and magazine advertising (questionnaire 2), including questions about whether they like reading advertisements and whether they believe the information in advertisements.

Participants were then given two vouchers for the 'inclass store', which could be redeemed for their choice of snack foods. The 'store' items included the three items advertised in the magazine (e.g. Cosmic brand lollipop); items that were the same in terms of size, taste and appearance, but not advertised brands (e.g. Sweetworld lollipop); and healthy foods (sultanas, fruit and rice wheels). The store selection was carefully designed to ensure both an equal number of 'healthy' and 'unhealthy' choices and an equivalence of portion sizes across the food choices. Once they had completed their purchases, participants answered questions about the food they chose and how healthy they thought their choices were (questionnaire 3), and then completed an unrelated task until the end of the lesson.

Finally, participants were given a 'parent questionnaire' to take home; this was in a sealed envelope and included an information sheet, the questionnaire itself and a replypaid envelope.

The study protocol was approved by the University's Ethics Committee and the NSW Department of Education and Training.

\section{Results}

\section{Children's responses}

A total of forty-seven children took part in the experimental study and completed the survey (twenty-five in the experimental condition and twenty-two in the control condition). There was a larger proportion of male children in the experimental group, but this difference was not statistically 
Table 1 Attitudes towards magazine advertising among children ( $n$ 47) aged 5-12 years, New South Wales, Australia

\begin{tabular}{|c|c|c|c|c|c|c|}
\hline & \multicolumn{2}{|c|}{ Yes } & \multicolumn{2}{|c|}{ Sometimes } & \multicolumn{2}{|c|}{ No } \\
\hline & $\%$ & $n$ & $\%$ & $n$ & $\%$ & $n$ \\
\hline I believe what they show in magazine advertisements & $8 \cdot 5$ & 4 & $63 \cdot 8$ & 30 & $27 \cdot 6$ & 13 \\
\hline I like magazine advertisements & $31 \cdot 9$ & 15 & $40 \cdot 4$ & 19 & $25 \cdot 5$ & 12 \\
\hline I think magazine advertisements are great & $25 \cdot 5$ & 12 & $44 \cdot 7$ & 21 & $29 \cdot 8$ & 14 \\
\hline When advertisements and promos are in magazines, I feel like reading the advertisements & $8 \cdot 5$ & 4 & $48 \cdot 9$ & 23 & $40 \cdot 4$ & 19 \\
\hline I think magazine advertisements are good & $34 \cdot 0$ & 16 & $34 \cdot 0$ & 16 & $29 \cdot 8$ & 14 \\
\hline I think that magazine advertisements tell the truth & $17 \cdot 0$ & 8 & $55 \cdot 3$ & 26 & $27 \cdot 6$ & 13 \\
\hline When advertisements and promos are in magazines, I enjoy reading the advertisements & $29 \cdot 8$ & 14 & $23 \cdot 4$ & 11 & $38 \cdot 3$ & 18 \\
\hline
\end{tabular}

significant $\left(68 \%\right.$ compared with $\left.41 \% ; \chi^{2}=0 \cdot 6\right)$. The participants were fairly evenly spread across year groups $(34 \cdot 0 \%$, Years $1-2 ; 27 \cdot 6 \%$, Years 3-4; and $36 \cdot 2 \%$, Years 5-6), and ranged in age from 5 to 12 years. Participant age did not differ significantly between conditions (mean age of 8.7 and 8.8 years for experimental and control groups, respectively). Of the forty-seven participants, twelve reported that they read some or most issues of Krash and fourteen that they read some or most issues of $\mathrm{D}$-Mag.

All variables were analysed by experimental condition and gender, and the results are reported for the sample as a whole, with any significant differences between groups being discussed.

\section{Food choice factors}

Taste was identified as the most important factor in choosing a snack food (with $59 \cdot 6 \%$ stating that this was very important and $27 \cdot 6 \%$ stating that it was important), and only $10 \cdot 6 \%$ stated that it was not important. The majority of participants reported that it was very important $(57 \cdot 4 \%)$ or important $(29 \cdot 8 \%)$ that the snack food be healthy, and very important $(40 \cdot 4 \%)$ or important $(23 \cdot 4 \%)$ that it be fun. Girls were more likely to state that it was important that a snack food be healthy $(t=1 \cdot 73, P=0 \cdot 003)$, and boys that it taste $\operatorname{good}(t=-1 \cdot 07, P=0 \cdot 031)$.

\section{Attitudes towards magazine advertising}

The participants showed generally positive attitudes towards magazine advertising, with the majority reporting that they like $(31.9 \%)$ or sometimes like $(40 \cdot 4 \%)$ magazine advertisements and that they do (29.8\%) or sometimes do (23.4\%) enjoy reading them (Table 1 ). However, they displayed a degree of scepticism towards advertising, with $63.8 \%$ stating that they sometimes believe and $27 \cdot 6 \%$ stating that they do not believe what they show in advertisements, and $55.3 \%$ stating that they sometimes think and $27 \cdot 6 \%$ that they do not think magazine advertisements tell the truth. There was no significant difference in the perceived believability of magazine advertising by age of participant, although younger participants were more likely to state that they like magazine advertisements $(F=4 \cdot 27$, $P=0 \cdot 001)$ and that they think magazine advertisements are great $(F=4 \cdot 82, P=0 \cdot 001)$. There were no differences on any of these items by gender.

\section{Food choices}

The children's food choices were categorized as 'advertised unhealthy food' (there were no healthy foods advertised in the magazines), 'non-advertised unhealthy food' (i.e. the matched food products) and 'healthy food'.

Three-quarters of the participants selected no healthy food items, and only one chose two healthy foods. A similar distribution was found for non-advertised unhealthy foods $(20 \cdot 8 \%$ chose one and $4 \cdot 2 \%$ chose two $)$. The majority of children selected one $(33 \cdot 3 \%)$ or two $(56 \cdot 3 \%)$ of the advertised foods. Participants in both groups were equally (un)likely to select a healthy food item; results showed a mean of 0.28 and 0.23 items/child in the experimental and control groups, respectively.

However, they differed in their choices of unhealthy food products, and these differences were statistically significant using a one-tailed test (which is appropriate given the a priori directional hypotheses). Those in the experimental group chose more advertised food items (with a mean of 1.64 compared with $1.32 ; t=1 \cdot 71$, $P=0 \cdot 046)$ and less of the non-advertised unhealthy food items (with a mean of $0 \cdot 16$ compared with $0 \cdot 45$; $t=-1 \cdot 88, P=0 \cdot 038$ ). It is particularly interesting to note that only one child in the 'advertising' condition chose no advertised foods (compared with four in the 'control' condition) and seventeen in the 'advertising' condition chose only advertised foods (compared with ten in the 'control' condition).

Given the age of the children participating in the present study, it was also important to assess whether participants understood the difference between 'healthy' and 'unhealthy' foods. A total of ninety-four food choices were made (two/ child), twelve were for healthy foods and eighty-two for unhealthy foods, and the respondents provided a classification for eighty-five of their ninety-four choices. Of the twelve healthy food choices, the relevant participant correctly classified ten as healthy foods and incorrectly classified one as unhealthy (one non-response); of the eighty-two unhealthy food choices, sixty-eight were correctly classified as unhealthy and six incorrectly classified as healthy (eight non-responses). Importantly, there was no statistically significant association between the stated importance of a snack food being healthy, tasty or fun and the actual choice of healthy or unhealthy foods. 


\section{Parent survey}

A total of twenty-four parents (51\%) completed and returned the parent survey: thirteen from the intervention group and eleven from the control group. The children ranged in age from 5 to 11 (mean $8 \cdot 3$ ) years. There were no significant differences between the two groups on any of the items, except one (described below); thus, the results are reported for the sample as a whole.

\section{Food consumption patterns}

The majority of parents (71\%) reported that their child consumed one or more pieces of fruit/d, and ate vegetables with dinner $\geq 5$ times/week (67\%). Most reported that their child ate cereal for breakfast 2-4 times (54\%) or 5-7 times/week (38\%). The parents reported that their children consumed takeaway food $<1$ (67\%) or $1-2$ times/week (29\%). The majority reported a less than weekly consumption of potato chips (63\%), with $29 \%$ reporting weekly and only $8 \%$ reporting daily consumption. Consumption of chocolate and lollies was more common, with $38 \%$ reporting a weekly consumption of each, and $38 \%$ and $33 \%$, respectively, a consumption of 2-6 times/week. In relation to packaged snacks, parents were asked separately about weekday and weekend consumption, with consumption $\geq 1$ time/d more common on weekdays (46\%) than on weekends (29\%). The majority of parents reported that their child drinks soft drink and cordial $<1$ time/week (63\% for both), although consumption daily or several times per day was commonly reported for cordial (29\%) but not by any parent for soft drink.

\section{Food requests}

Children were described as often (54\%) or sometimes (33\%) asking for fruit and vegetables and often (54\%) or sometimes (33\%) for dairy (although the latter may include less-healthy dairy-based foods). Requests for chocolates and confectionery were more likely to be described as occurring sometimes (67\%) than as often (33\%), as were requests for packaged snacks (54\% and $33 \%)$ and takeaway foods (67\% and 29\%). Parents were asked what they thought influenced the food requests made by their children; only $25 \%$ thought that these requests were often influenced by parents and $21 \%$ by friends, ${ }^{*}$ but $58 \%$ thought that the influence was often advertising.

When asked whether they felt pressurized to buy certain types of foods for their children, 25\% said that they often felt pressurized to buy fruit and vegetables and $54 \%$ said dairy, with the source of the 'pressure' equally likely to be friends or family (17\%), the child (13\%) or

\footnotetext{
* This was the only item in the parent survey for which responses differed by group, with parents in the experimental group more likely to report that the influence is 'often' friends and those in the control group more likely to report that it is 'sometimes' friends $\left(\chi^{2}=0 \cdot 036\right)$.
}

advertising (17\%). Parents were less likely to report often feeling pressurized to buy chocolate and confectionery (13\%), packaged snacks (13\%) or takeaway meals (8\%), although approximately half said that they 'sometimes' felt this way. The source of the 'pressure' was much clearer in this case, with $46 \%$ reporting 'often' being pressurized by their children, $13 \%$ by advertising and none by family or friends. It is also interesting to note that two parents hand-wrote on the survey form that they felt pressurized by 'sports advertising/sports sponsors'.

\section{Feasibility of the study design}

The primary aim of the present study was to examine the feasibility of the study design for assessing the effects of food advertising on children's food choices. Thus, where the study protocol required a decision between elements that could lead to demand effects, wherever possible we erred on the side of decisions that would favour the null hypothesis. For example, questions regarding factors that influence food choice could have been asked before reading the magazine or after reading the magazine; we chose the former in order to maximize the time and number of distracter activities between responding to those items and making the actual food choices. Similarly, we could have asked the questions regarding attitudes to advertising in general before the food choices or after the food choices; we elected to do the former as again this decision would have favoured the null hypothesis - as it would have reduced the likelihood of those in the 'advertising' condition selecting the advertised foods. However, we asked the questions about liking and perceived healthiness of the products in the 'store' after the behavioural measure as we felt that this was likely to influence food choices.

Importantly, we found that the study protocol was acceptable to the children, parents and care providers; that the children participated actively in the activities; and that the experimental 'store' functioned effectively to enable the children to make actual food choices.

\section{Discussion}

Our study adds to the existing literature on the role of food advertising in influencing children's food choices in several ways. First, it builds on the existing (although small) body of literature on food advertising to children through the print media. Previous studies have examined the extent and nature of food advertising in children's magazines ${ }^{(35,36,38)}$, and children's interpretation of this advertising $^{(39)}$, but to date there is no published research on children's behavioural responses to child-targeted print advertising, other than a single study that had children read advertisements in isolation from the context of their magazine ${ }^{(37)}$. Second, it provides further support for the body of research showing that food advertising does affect 
children's food choices ${ }^{(16,29,31)}$, by showing that these effects are not unique to TV advertising. Third, it confirms that self-reported intention does not necessarily predict actual behaviour - which is particularly important in the study of behaviour such as food choices in which social desirability effects are likely to result in under-reporting of 'bad' choices - and shows the need for studies to include actual behaviour in order to accurately assess the effects of advertising. Finally, it provides a simple, easily replicable methodology for testing the behavioural effects of exposure to print (and other media) food advertising.

Despite the small sample size in this feasibility study, we found that exposure to magazine advertising influenced food choices, with those exposed to magazines containing food advertisements more likely to choose the advertised foods than those reading magazines with no food advertisements. Advertising has been described as 'subverting power relations between adults and children' ${ }^{, 40)}$. Our finding that children chose unhealthy foods when given the opportunity to do so (when not observed by their parents), combined with parent respondents' reports of feeling pressurized by their children, suggests that it may indeed be the case that advertising alters the perceived power balance. Further studies could investigate this idea by, for example, conducting a similar experiment with parents present.

\section{Attitudes to food advertising}

It was perhaps not surprising that the majority of participants reported that they like magazine advertisements, think they are 'great' and enjoy reading them. A recent review of food advertising to children across twenty European Union countries found that commonly used creative strategies included references to children's movies and movie characters; appeals to play, fun, action and adventure; and use of humour, fantasy, cartoon characters and celebrities ${ }^{(41)}$.

It was encouraging to note that over a quarter of participants stated that they do not believe what is shown in magazine advertisements and do not think that advertisements tell the truth (and over half think that this is sometimes the case). However, this finding should be interpreted with caution. Only four participants said that they always believe what is shown in advertisements and only eight said that advertisements always tell the truth, suggesting that they believe they display a degree of scepticism in their responses to advertising. However, given that many of them stated that they like reading advertisements and that previous research shows an association between liking advertisements and food choices (although these previous studies focused on TV advertising), it is possible that these messages may work to undermine that scepticism and thus have a subconscious effect on food choices. Further, a recent study with 269 children aged 8-12 years concluded that, although cognitive defences (advertising recognition and awareness of the persuasive intent of advertising) reduced the impact of advertising on desire for the product among those aged 10-12 years, among younger children, this awareness actually increased the impact of advertising ${ }^{(42)}$.

\section{Influences on food choices}

It is interesting to note that, although the majority $(87 \cdot 2 \%)$ of participants stated it was important or very important for the snack foods they chose to be healthy, only one chose two healthy food items from the in-class store, and ten chose one. That is, despite the stated centrality of 'healthiness' in snack food choice, only less than onequarter chose even one healthy item when placed in an actual choice situation. The data show that the children could differentiate between healthy and unhealthy foods, and therefore these choices were not the result of a lack of awareness of the 'healthiness' of their choices. Further, the fact that there was no statistical association between the stated importance of 'healthy' snack foods and the actual food choices suggests that, when asked directly, children are likely to report socially desirable responses (i.e. those their parents, teachers or other adults 'want' to hear) rather than their actual preferences. This is consistent with previous research, which has shown that parents' reports of behaviours and intentions regarding the provision of healthy foods for their children are influenced by perceptions of value judgements associated with these food choices ${ }^{(43)}$. The fact that children (and adults) differ in what they say they do and their actual behaviour shows the value of using an experimental design such as that in the present study.

The parent respondents clearly identified advertising as a key influence on their children's food purchase requests, with over half stating that advertising was 'often' the influencing factor. Given the high proportion of child-targeted advertising for high-fat, high-sugar foods, it is not surprising that almost half of the parents reported 'often' being pressurized by their children - but never by their family or friends - to purchase unhealthy snack foods, and 13\% 'often' feeling pressurized by advertising to buy high-fat/ high-sugar foods. It is also interesting to note that $17 \%$ of parents reported 'often' feeling pressurized by advertising to buy dairy foods, which are less frequently advertised to children; it is possible that this relates to the messages inherent in some food advertisements that 'good parents' feed their children certain foods, but future research could investigate this perception more thoroughly.

\section{Limitations}

The sample size (forty-seven children and twenty-four parents) limited our ability to conduct more complex analysis. For example, it was not possible to test relationships between attitudes to advertising and selection of advertised food items with the sample of twenty-five children in the 'advertising' condition, given that only one child did not choose an advertised food and eighteen 
chose two advertised foods. There were also limitations stemming from the use of vacation care centres as a study venue; participants spanned a range of ages and literacy levels within each group, and the lack of a physical and discipline structure meant that it was difficult to prevent participants from observing and interacting with each other during the shopping task. The latter would potentially have had a significant impact on food choices, given the importance of peer approval in this age group and the social nature of the setting. However, it is likely that this would have resulted in an underestimation of the effects of advertising, as the students in the control group, although not exposed to the advertisement, would have observed their peers from the other group choosing the advertised foods. Similarly, having children answer questions about influences on their snack food choices may have primed them to make healthier food choices; however, again, this would most likely result in an underestimation of the effects of advertising.

The physical limitations imposed by the location of the data collection (i.e. vacation care facilities that do not have appropriate facilities for physical assessment), combined with the lack of validity of self-reported weight and height among children in this age group, meant that we did not assess the weight or BMI of the study participants. Given previous research suggesting that overweight children respond differently to food advertisements than normalweight children, future research could usefully include objective measures of participant BMI.

We did not include measures of determining whether the children could differentiate between an advertisement and other magazine content, as the study design already required a significant investment of time and energy from the children and adding further measures would have been problematic. However, our previous work in this area suggests that children are not always aware of the persuasive intent $^{(39)}$. Further, our work and that of others suggests that the distinction between advertisements and editorial content in children's magazines is even more problematic than for $\mathrm{TV}^{(38)}$; for example, a recent study found that ten marketing academics could not agree whether selected pages from children's magazines were advertisements or product placements ${ }^{(45)}$. However, future studies could usefully measure participants' ability to differentiate between advertisements and editorial content and assess whether this affects food choices.

\section{Conclusions}

This was a small-scale study, in one geographical area, and thus the results cannot be generalized to broader populations of children. However, this was a feasibility study, and the first to directly assess the effects on children of exposure to food advertising within the context of reading a magazine targeted at their age group. Importantly, the present study shows that the methodology is feasible, with a good response rate from both child and parent participants, and positive feedback from children, parents and centres regarding their involvement in the study. Further, even with this small sample size and the acknowledged methodological limitations of the study venues, we found the predicted effect of exposure to this form of advertising on food choices. Future research could use this methodology with larger and more representative samples, preferably in a school-based environment, which would provide more homogeneous samples in terms of age and reading ability. A replication of our findings - given the growing number of children's magazines in many countries and the blurring of lines between advertisements and editorial content in these publications - would suggest an urgent need for improved monitoring and regulation of advertising to children in this very targeted and under-researched medium.

\section{Acknowledgements}

The present study was part of an ongoing research project funded by the Australian Research Council. The authors have no conflict of interest to declare. S.C.J. and L.K. designed the study protocol, developed the data collection instruments and finalized the manuscript for submission. L.K. conducted the intervention. S.C.J. analysed the data and drafted the manuscript. The authors thank Kelly Andrews, Belinda Fabrianesi and Nadia Mannino from the Centre for Health Initiatives, and Jessica Mantei from the Faculty of Education, University of Wollongong, for their assistance with the data collection and project management. They also thank the participating centres, children and parents for their contribution to the present study.

\section{References}

1. Jackson-Leach R \& Lobstein T (2006) Estimated burden of paediatric obesity and co-morbidities in Europe. Part 1. The increase in the prevalence of child obesity in Europe is itself increasing. Int J Paediatr Obes 1, 26-32.

2. Lobstein T \& Jackson-Leach R (2007) Child overweight and obesity in the USA: prevalence rates according to USA definitions. Int J Paediatr Obes 2, 62-64.

3. Harris JL, Pomeranz JL, Lobstein T et al. (2009) A crisis in the marketplace: how food marketing contributes to childhood obesity and what can be done. Annu Rev Public Health 30, 211-225.

4. Margarey A, Daniels L \& Boulton T (2001) Prevalence of overweight and obesity in Australian children and adolescents; reassessment of 1985 and 1995 data against new standard international definitions. Med J Aust 174, 561-564.

5. Australian Bureau of Statistics (2009) 4364.0 - National Health Survey: Summary of Results, 2007-2008 (Reissue): Risk Factors. Canberra: ABS.

6. Hampl JS, Taylor CA \& Johnston CS (1999) Intakes of vitamin C, vegetables and fruits: which schoolchildren are at risk? J Am Coll Nutr 18, 582-590.

7. Skinner JD, Betty RC, Houck KS et al. (1999) Longitudinal study of nutrient and food intakes of white preschool children aged 24 to 60 months. J Am Diet Assoc 99, 1514-1521. 
8. Fitzgibbon ML \& Stolley M (2006) Promoting health in an unhealthful environment: lifestyle challenges for children and adolescents. J Am Diet Assoc 106, 518-522.

9. Magnus A, Haby MM, Carter R et al. (2009) The costeffectiveness of removing television advertising of high-fat and/or high-sugar food and beverages to Australian children. Int J Obes (Lond) 33, 1094-1102.

10. Vereecken CA, Keukelier E \& Maes L (2004) Influence of mother's educational level on food parenting practices and food habits of young children. Appetite 43, 93-103.

11. Variyam J, Blaylock J, Lin B et al. (1999) Mother's nutrition knowledge and children's dietary intakes. Am J Agric Econ 81, 373-384.

12. Wetter A, Goldberg J, King A et al. (2001) How and why do individuals make food and physical activity choices? Nutr Rev 59, 3 Pt 2, S11-S20.

13. Kunkel D (2001) Children and television advertising. In Handbook of Children and Media, pp. 375-393 [D Singer and J Singer, editors]. Thousand Oaks, CA: Sage.

14. Calvert SL (2008) Children as consumers: advertising and marketing. Future Child 18, 205-234.

15. Kotz K \& Story M (1994) Food advertisements during children's Saturday morning television programming: are they consistent with dietary recommendations? J Am Diet Assoc 94, 1296-1300.

16. Hastings G, Stead M, McDermott L et al. (2003) Review of Research on the Effects of Food Promotion to Children. London: Food Standards Agency.

17. Lemos DMP (2004) Saturday morning children television food advertising ... The nightmare of nutrition educators. J Pediatr Gastroenterol Nutr 39, Suppl. 1, S471-S472.

18. Maryam A, Mehdi M-R, Masood K et al. (2005) Food advertising on Iranian children's television: a content analysis and an experimental study with junior high school students. Ecol Food Nutr 44, 123-133.

19. Wilson N, Signal L, Nicholls S et al. (2006) Marketing fat and sugar to children on New Zealand television. Prev Med 42, 96-101.

20. Kelly B, Smith B, King L et al. (2007) Television food advertising to children: the extent and nature of exposure. Public Health Nutr 10, 1234-1240.

21. Utter J, Scragg R \& Schaaf D (2006) Associations between television viewing and consumption of commonly advertised foods among New Zealand children and young adolescents. Public Health Nutr 9, 606-612.

22. Buijzen M, Schuurman J \& Bomhof E (2008) Associations between children's television advertising exposure and their food consumption patterns: a household diary-survey study. Appetite 50, 231-239.

23. Klepp K-I, Wind M, de Bourdeaudhuij I et al. (2007) Television viewing and exposure to food-related commercials among European school children, associations with fruit and vegetable intake: a cross sectional study. Int $J$ Behav Nutr Phys Act $\mathbf{4}, 46$.

24. O'Dougherty M, Story M \& Stang J (2006) Observations of parent-child co-shoppers in supermarkets: children's involvement in food selections, parental yielding, and refusal strategies. J Nutr Educ Behav 38, 183-188.

25. Brooke F \& Kelly P (2009) Dolly girls: tweenies to artifacts of consumption. J Youth Stud 12, 599-613.

26. Chou S-Y, Rashad I \& Grossman M (2008) Fast-food restaurant advertising on television and its influence on childhood obesity. J Law Econ 51, 599-618.
27. Zimmerman FJ \& Bell JF (2010) Associations of television content type and obesity in children. Am J Public Health 100, 334-340.

28. Cairns G, Angus K \& Hastings G (2009) The Extent, Nature and Effects of Food Promotion to Children: A Review of the Evidence to December 2008. Geneva: WHO.

29. Committee on Food Marketing and the Diets of Children and Youth (2006) Food Marketing to Children and Youth: Threat or Opportunity? [JM McGinnis, J Gootman and VI Kraak, editors]. Washington, DC: National Academies Press.

30. Story M \& French S (2004) Food advertising and marketing directed at children and adolescents in the US. Int J Behav Nutr Phys Act 1, 3.

31. UK Office of Communication (2004) Childhood Obesity Food Advertising in Context. Children's Food Choices, Parents' Understanding and Influence, and the Role of Food Promotion. London: Ofcom.

32. Soldow GF (1983) The processing of information in the young consumer. $J$ Advert 12, 4-14.

33. Stoneman Z \& Brody GH (1983) Immediate and long-term recognition and generalization of advertised products as a function of age and presentation mode. Dev Psychol 19, 56-61.

34. Curtis J (2004) Small targets. Marketing, 19 May, p. 38.

35. Kelly B \& Chapman K (2007) Food references and marketing to children in Australian magazines: a content analysis. Health Promot Int 22, 284-291.

36. Cowburn G \& Boxer A (2007) Magazines for children and young people and the links to Internet food marketing: a review of the extent and type of food advertising. Public Health Nutr 10, 1024-1031.

37. King L \& Hill AJ (2008) Magazine adverts for healthy and less healthy foods: effects on recall but not hunger or food choice by pre-adolescent children. Appetite 51, 94-197.

38. Jones SC \& Reid A (2010) Children's magazines: reading resources or food marketing tools? Public Health Nutr 13, 393-399.

39. Jones SC, Mannino N \& Green J (2010) 'Like me, want me, buy me, eat me': relationship-building marketing communications in children's magazines. Public Health Nutr $\mathbf{1 3}$ 2111-2118.

40. Kenway J \& Fitzclarence L (1999) Designing generations: hybridising entertainment, advertising and education. Aust J Educ 43, 300-316.

41. Matthews AE (2008) Children and obesity: a pan-European project examining the role of food marketing. Eur J Public Health 18, 7-11.

42. Rozendaal E, Buijzen M \& Valkenburg P (2009) Do children's cognitive advertising defenses reduce their desire for advertised products? Communications 34, 287-303.

43. Jones SC, McVie D \& Noble GI (2008) You are what your children eat: using projective techniques to investigate parents' perceptions of the food choices parents make for their children. Open Commun J 2, 23-28.

44. Jones SC \& Fabrianesi B (2008) Gross for kids but good for parents: differing messages in advertisements for the same products. Public Health Nutr 11, 588-595.

45. Acharya DS \& Mizerski R (2005) Expert opinion on the content and intention of material from a magazine targeted to 7 to 10 year old beginning readers. Australian and New Zealand Marketing Academy Conference, pp. 1-7. http:// smib.vuw.ac.nz:8081/www/anzmac2005/cd-site/authors1. html/ (accessed July 2010). 